\title{
The Role of an Inert Electrode Support in Plasmonic
}

\section{Electrocatalysis}

Sagar Ganguli and Alina Sekretareva*

Department of Chemistry, Ångström Laboratory, Molecular Biomimetics, Uppsala University, 75120, Uppsala, Sweden

Keywords: Plasmonic electrocatalysis, benzyl alcohol oxidation, graphite electrode support, gold nanoparticles, charge transfer kinetics

\begin{abstract}
Plasmonic nanostructures loaded onto catalytically inert conductive support materials are believed to be advantageous for maximizing photocatalytic effects in photoelectrochemical systems due to the increased efficiency of Schottky barrier-free architectures in collecting hot charge carriers. However, the systematic mechanistic investigation and description of the inert electrode support contribution to plasmonic electrocatalysis is missing. Herein, we systematically investigated the effect of the supporting electrode material on the observed photocatalytic enhancement by comparing photoelectrocatalytic properties of AuNPs supported on highly oriented pyrolytic graphite (HOPG) and on indium tin oxide (ITO) electrodes using electrocatalytic benzyl alcohol $(\mathrm{BnOH})$ oxidation as a model system. Upon illumination, only $\sim(3 \pm 1) \%$ enhancement in catalytic current was recorded on the AuNP/ITO electrodes in contrast to $\sim(42 \pm 6) \%$ enhancement on AuNP/HOPG electrodes. Our results showed that the local heating due to light absorption by the electrode material itself independent of localized surface plasmon effects is the primary source of the observed significant photo-induced enhancement on the HOPG electrodes in comparison to the ITO electrodes. Moreover, we
\end{abstract}


demonstrated that an increased interfacial charge transfer at elevated temperatures, and not faster substrate diffusion is the main source of the enhancement. This work highlights the importance of systematic evaluation of contributions of all parts, even if they are catalytically inert, to the light-induced facilitation of catalytic reactions in plasmonic systems.

\section{Introduction}

Plasmonic nanostructures $(\mathrm{Au}, \mathrm{Ag}, \mathrm{Cu})$ are being increasingly researched as attractive materials for facilitating light-driven heterogeneous catalysis. ${ }^{1}$ Interaction of the noble metal nanostructures with an incident electromagnetic wave of resonant frequency results in collective oscillation of the confined electrons known as localized surface plasmons (LSPs). ${ }^{2}$ Non-radiative rapid decay of LSPs through Landau damping leads, first, to the generation of hot charge carriers (electrons and holes) inside the nanostructures within 100 fs. These hot carriers further thermalize inducing a local temperature increase. ${ }^{3}$ Both these effects, as well as enhanced electric fields near the surface of nanostructures due to LSPs, can contribute to the enhancement of chemical transformation rates in photocatalytic systems based on plasmonic nanostructures. ${ }^{4}$ Moreover, facilitation of several electrocatalytic reactions, such as alcohol oxidation, water splitting, and carbon dioxide reduction, by LSP effects has been reported. ${ }^{5,6}$

In electrocatalytic systems, the plasmonic nanostructures are commonly loaded on support materials to form plasmonic photoelectrodes. The frequently used support materials can be broadly classified into two types: (i) semiconductors, which form a Schottky barrier at the contact with the metallic nanostructures, and (ii) conductive supports that form the continuous energy level. Photoelectrodes free of the Schottky barrier are considered to be more promising for maximizing photocatalytic effects, as it is believed that the Schottky barrier limits the collection efficiency of hot charge carriers. ${ }^{7}$ Among conductive supports, carbon-based materials (glassy carbon (GC), graphene, graphite), and indium tin oxide (ITO) are the most frequently used in Schottky barrier-free plasmonic systems due to their catalytic inertness. 
Despite the great interest in designing the Schottky barrier-free systems with maximized hot carrier utilization, the role of the catalytically-inert electrode support in photocurrent enhancement is discussed only in a few reports. For example, Shi et al. reported that the photothermal contribution to the photocurrent of oxygen reduction reaction on $\mathrm{Ag}$ nanostructures could be minimized by using graphene as a support. ${ }^{8}$ Liao and co-workers attributed the enhancement of glucose electro-oxidation under irradiation on a reduced graphene oxide (rGO)-AuNP GC composite electrode to the excellent electron transfer capability of rGO facilitating hot charge carrier separation. ${ }^{9}$ Kim's group proposed that rGO contributes to significant enhancement of photocatalytic activity of AuNPs/Pd/rGO towards oxygen evolution and hydrogen evolution reactions by mediating hot-electron transfer to catalytic sites on Pd. ${ }^{10}$ All these reports suggest a positive effect of carbon-based materials, particularly with a $\mathrm{sp}^{2}$ carbon structural motif, on the charge separation process leading to increased photocurrents in electrocatalytic systems. However, the systematic mechanistic investigation and description of the inert electrode support contribution to plasmonic electrocatalysis is missing.

Here, we systematically investigate the effect of the supporting electrode material on the observed photocatalytic enhancement and its role in facilitating charge separation at illuminated electrodes. We compare photoelectrocatalysis by AuNPs supported on highly oriented pyrolytic graphite (HOPG), a sp² carbon-based material with a multilayer graphene structure with minimum defects, ${ }^{11}$ and on ITO using electrocatalytic benzyl alcohol $(\mathrm{BnOH})$ oxidation as a model system. Employing voltammetry (linear sweep (LSV) and cyclic (CV)), photocurrent measurements at various wavelengths, temperature variation studies, and hydrodynamic voltammetry, we show that the local heating due to light absorption by the electrode material itself independent of LSP effects is the primary source of the observed significant photoinduced enhancement on the carbon-based electrodes in comparison to ITO. Moreover, we 
demonstrate that an increased interfacial charge transfer at elevated temperatures, and not faster substrate diffusion is the main source of the enhancement. This work highlights the importance of systematic evaluation of contributions of all parts, even if they are catalytically inert, to the light-induced facilitation of catalytic reactions in plasmonic systems.

\section{Materials}

AuNPs (20 nm diameter; OD 1, stabilized suspension in $0.1 \mathrm{mM}$ PBS, reactant free), $\left[\mathrm{Ru}\left(\mathrm{NH}_{3}\right)_{6}\right] \mathrm{Cl}_{2}$, and $\mathrm{BnOH}$ were purchased from Sigma Aldrich. Sodium carbonate $\left(\mathrm{Na}_{2} \mathrm{CO}_{3}\right)$, and sodium bicarbonate $\left(\mathrm{NaHCO}_{3}\right)$ were procured from Merck. HOPG rod (radius: $\left.0.1 \mathrm{~cm}\right)$ was purchased from Goodfellow and embedded in a Teflon sheath keeping only the top surface exposed. Both counter (Pt ring) and reference $(\mathrm{Ag} / \mathrm{AgCl} ; 1(\mathrm{M}) \mathrm{KCl})$ electrodes were purchased from $\mathrm{CH}$ Instruments. An ITO-covered glass $\left(8-12 \Omega / \mathrm{cm}^{2}\right)$ was procured from Redoxme AB. The green $\left(325 \mathrm{~mW} / \mathrm{cm}^{2}, 532 \mathrm{~nm}\right)$, blue $\left(206 \mathrm{~mW} / \mathrm{cm}^{2}, 405 \mathrm{~nm}\right)$ and red $\left(266 \mathrm{~mW} / \mathrm{cm}^{2}, 650\right.$ $\mathrm{nm}$ ) lasers were purchased from Lucinda. Carbon coated copper TEM grid was procured from Agar Scientific.

\section{Experimental Method}

Transmission electron microscope (TEM) measurements were carried out using FEI Tecnai F30 ST $300 \mathrm{kV}$ field emission gun TEM/STEM instrument equipped with a Gatan Imaging Filter, energy-dispersive detector, high-angle annular dark-field detector, Lorentz lens, and Bi-prism for Electron Holography. The TEM samples were prepared by depositing a drop of the asreceived AuNPs suspension on a carbon-coated copper TEM grid and dried at room temperature for several hours before examination in the TEM.

Uv-Vis absorption spectroscopy measurements: First, the as-received AuNPs suspension was diluted 100 times with deionized water. The resulting suspension was then transferred to a cuvette with a path length of $1 \mathrm{~cm}$ and spectroscopic measurements were carried out in the 
medium-scan mode of Cary Varian 50 Bio UV-Visible Spectrophotometer. The wavelength range and slit-width were $800-300 \mathrm{~nm}$, and $3 \mathrm{~nm}$, respectively. For solid-state measurements, $30 \mu \mathrm{L}$ of as-received AuNPs solution was first dropcasted on a cover-slip followed by drying under ambient conditions. Thereafter, the coverslip was placed in the light path of the spectrometer using a holder, and measurements were performed with the same parameters.

Electrode Preparation: $5 \mu \mathrm{L}$ AuNPs suspension was carefully dropcasted on a polished HOPG (radius: $0.1 \mathrm{~cm}$; embedded in Teflon) electrode. It was then left overnight for drying to obtain AuNPs coated HOPG (AuNP/HOPG) electrode. A similar protocol was followed to develop AuNPs coated ITO electrodes (AuNP/ITO), where $40 \mu \mathrm{L}$ AuNP suspension was dropcasted on $0.5 \mathrm{~cm}^{2}$ of ITO coated glass. Thereafter, a non-conductive resin was applied on AuNP/ITO electrodes leaving exposed an area corresponding to the laser diameter. This ensured that the obtained electrochemical data is not convoluted from any background current.

Electrochemical Measurements: A three-electrode cell setup using AuNP/HOPG or AuNP/ITO as working, $\mathrm{Pt}$ as counter, and $\mathrm{Ag} / \mathrm{AgCl}(1(\mathrm{M}) \mathrm{KCl})$ as reference electrodes was employed to perform electrochemical measurements. $50 \mathrm{ml} 0.1$ (M) carbonate-bicarbonate buffer ( $\mathrm{pH} \sim 10.6$ ) was used as the electrolyte. The electrochemical experiments were performed using Gamry Reference 600 and Ivium CompactStat.h electrochemical workstations. Prior to performing electrocatalytic $\mathrm{BnOH}$ oxidation measurements, the AuNP/HOPG electrode was subjected to 15 cycles of $\mathrm{CV}$ at $50 \mathrm{mV} / \mathrm{s}$ between $0.45 \mathrm{~V}$ to $1.5 \mathrm{~V}$ vs RHE to obtain a kinetically stable state. Thereafter, $100 \mu \mathrm{L} \mathrm{BnOH}$ was added to the electrolyte and stirred till its complete dissolution to make $20 \mathrm{mM} \mathrm{BnOH}$ solution. In this solution, the electrode was again subjected to 15 cycles of $\mathrm{CV}$ in the same potential range at $50 \mathrm{mV} / \mathrm{s}$ before conducting measurements for data interpretation. Linear sweep voltammetry $(\mathrm{LSV}) / \mathrm{CV}$ were performed in the same potential window at $50 \mathrm{mV} / \mathrm{s}$ inside a Faraday cage under dark and light to evaluate the electrocatalytic performance. 


\section{Result and Discussion}

\section{Characterization of AuNPs}

Reactant-free AuNPs with an average size of $(18 \pm 4) \mathrm{nm}$ as characterized by TEM measurements (Figure 1A) have been used throughout this study. The AuNPs suspension showed a single, sharp absorption band with an absorption maximum at $518 \mathrm{~nm}$ (Figure 1B, orange trace). This is consistent with the dipole LSP resonance band of free electrons in spherical AuNPs. ${ }^{12}$ The additional absorption features at lower $(<470 \mathrm{~nm})$ wavelengths can be attributed to inter-band transitions in AuNPs. ${ }^{13}$ The AuNPs adsorbed on a solid support displayed a broad absorption feature covering the entire visible range with a maximum at around $627 \mathrm{~nm}$ along with the interband transitions at lower wavelengths (Figure 1B, grey trace). This broadening can be attributed to the near-field interaction between neighboring AuNPs as their proximity increases under solid-state conditions. ${ }^{14,15}$
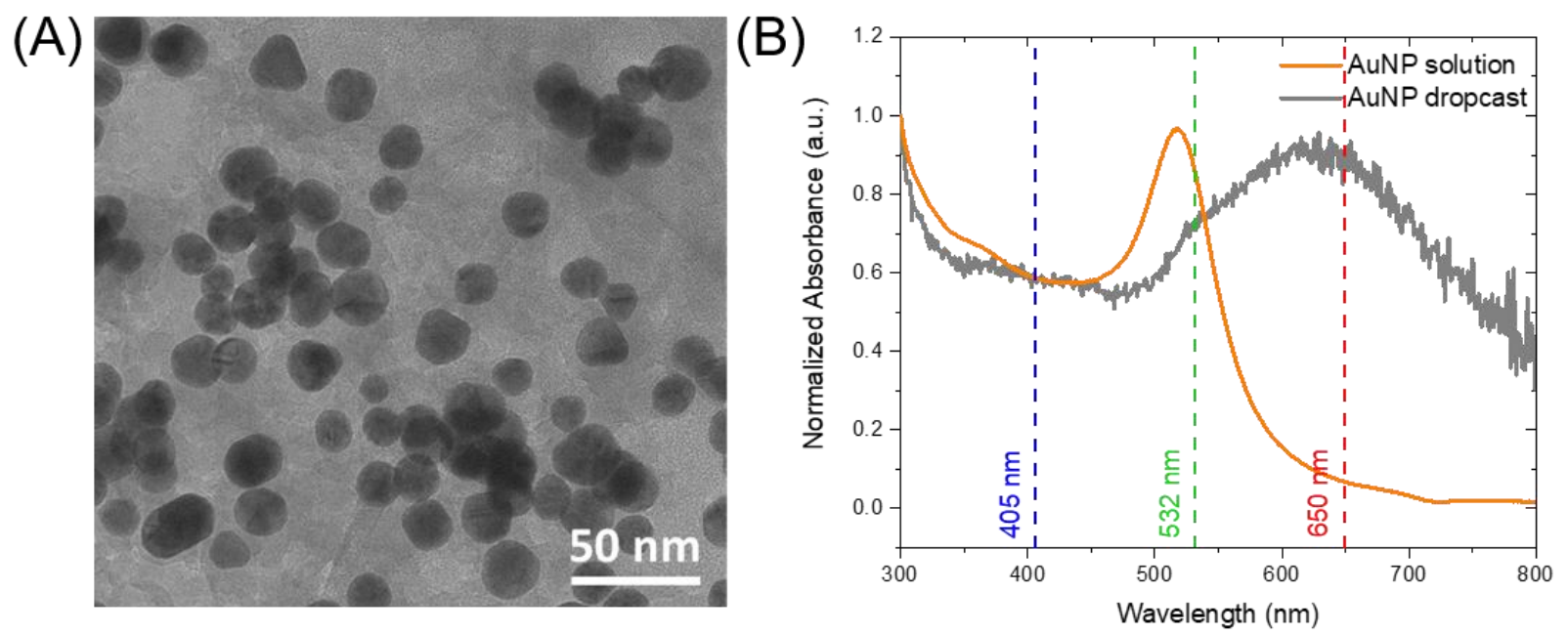

Figure 1: (A) TEM image of as-received reactant-free AuNPs with an average particle size of (18 \pm 4$) \mathrm{nm}$. (B) Uv-Vis absorption spectroscopy measurements of AuNP suspension (orange trace) and dried AuNPs dropcasted on a cover-slip (grey trace). The dashed lines indicate the wavelengths of the lasers used in plasmonic electrocatalysis (vide infra). 
The electrocatalytic activity of AuNPs supported on HOPG and ITO electrodes towards BnOH oxidation in the dark and under continuous-wave illumination using a selected laser excitation source light was investigated. The AuNPs-modified electrodes served as a working electrode in a three-electrode setup. The entire catalyst layer was illuminated by a light source placed at the bottom or at the front of the transparent cell for the AuNP/HOPG (Figure S1) and AuNP/ITO electrodes, respectively. Figures 2A and 2D show LSVs recorded on the AuNP/HOPG and AuNP/ITO electrodes, respectively, in $20 \mathrm{mM} \mathrm{BnOH}$ solution in the dark (black curves) and under irradiation with a $532 \mathrm{~nm}$ laser with the intensity of $325 \mathrm{~mW} / \mathrm{cm}^{2}$ (green curves). A distinct oxidation peak was observed on both electrodes in the dark (at 1.24 $\mathrm{V}$ vs RHE for AuNP/HOPG and at $1.15 \mathrm{~V}$ vs RHE for AuNP/ITO). Bare HOPG and ITO electrodes did not demonstrate significant catalytic activity towards $\mathrm{BnOH}$ oxidation compared to the AuNPs-modified electrodes (Figures S2 and S3, respectively). Thus, the observed anodic peaks were ascribed to $\mathrm{BnOH}$ oxidation on the AuNPs serving as catalytic centers. ${ }^{16}$

Upon illumination, a striking difference in the photocurrent responses was observed for the AuNPs supported on the HOPG and the ITO electrodes. Only $\sim(3 \pm 1) \%$ peak current enhancement upon irradiation with the $532 \mathrm{~nm}$ laser $\left(325 \mathrm{~mW} / \mathrm{cm}^{2}\right)$ was recorded on the AuNP/ITO electrodes in $20 \mathrm{mM} \mathrm{BnOH}$ solution in contrast to $\sim(42 \pm 6) \%$ enhancement on the AuNP/HOPG electrodes (Figures 2A, D). To understand the primary reason for the observed difference in the photocurrent responses and the source of the significant enhancement on the HOPG electrodes, we performed chronoamperometry measurements under alternating illumination (532 $\mathrm{nm}$ laser $\left.\left(325 \mathrm{~mW} / \mathrm{cm}^{2}\right)\right)$ at a fixed applied potential of $1.16 \mathrm{~V}$ vs RHE. Prior to switching on illumination, the electrodes were kept at the fixed potential to establish a stable dark current. Figures 2B-C show the photocurrent response of the AuNP/HOPG electrode in $20 \mathrm{mM} \mathrm{BnOH}$ solution. Upon switching on illumination (green areas in Figures 2B-C) an 
increase of the anodic current is observed. Subsequent switching of the light source (grey areas in Figures 2B-C) results in a decrease of the current back to the dark value. Both an increase of the photocurrent upon irradiation and a subsequent decrease when the light was off were gradual lasting over $20 \mathrm{~s}$ before reaching a steady-state value.

The photocatalytic enhancement of alcohol oxidation on plasmonic nanostructures supported on carbon-based materials has been previously attributed to the participation of hot charge carriers generated during plasmon decay. ${ }^{17-19}$ The lifetime of hot carriers generated due to Landau damping of the plasmons in AuNPs does not exceed one picosecond (ps)., ${ }^{3,20,21}$. Thus, for the photocurrent resulting from the participation of hot carriers in the catalytic reaction, one would expect a sharp decrease of the signal upon switching off illumination limited only by the time constant $(\tau)$ of the electrochemical cell. The time constant $(\tau)$ of the cell, defined as a product of the cell resistance $\left(\mathrm{R}_{\mathrm{s}}\right)$ and the double-layer capacitance $\left(\mathrm{C}_{\mathrm{dl}}\right)$, represents the minimum time the cell requires to respond to a perturbation and can be readily measured. ${ }^{22}$ To measure $\tau$, first, a potential in the non-Faradaic region ( $0.65 \mathrm{~V}$ vs RHE) was held for $20 \mathrm{~s}$ to reach a steady-state current followed by applying a small potential step $(10 \mathrm{mV}, 20 \mathrm{mV}$, and 30 $\mathrm{mV}$, respectively) (Figures S4A-C). This potential step (E) led to a sharp increase in the current corresponding to the charging of the double layer. This charging current thereafter decays according to Eqn. 1:

$i=\frac{E}{R_{S}} \cdot e^{-\frac{t}{R_{S} C_{d l}}}$

(Eqn. 1),

where $i$ and $t$ are current, and time, respectively. The time required for the current to reach $\sim 37 \%$ of its initial value represents the $\tau$ of the electrochemical system. ${ }^{22}$ The obtained value of the $\tau$ for the AuNP/HOPG electrodes is $\sim 30$ milliseconds (Figures S4A-C). The slow decay of the $\mathrm{BnOH}$ oxidation photocurrent on the AuNP/HOPG electrodes (Figures 2B-C) over a time greater than the five cell time constants $(5 \tau)$ undermines the direct participation of hot carriers 
in the catalytic reaction. This is in agreement with the recently reported by Nocera et al. finding that ps-range lifetimes of excited states are orders of magnitude shorter than that required for productive photochemistry. ${ }^{23}$ Thus, the observed slow decay of the photocurrent suggests that heating is the primary source of the photocurrent on the AuNP/HOPG electrodes.
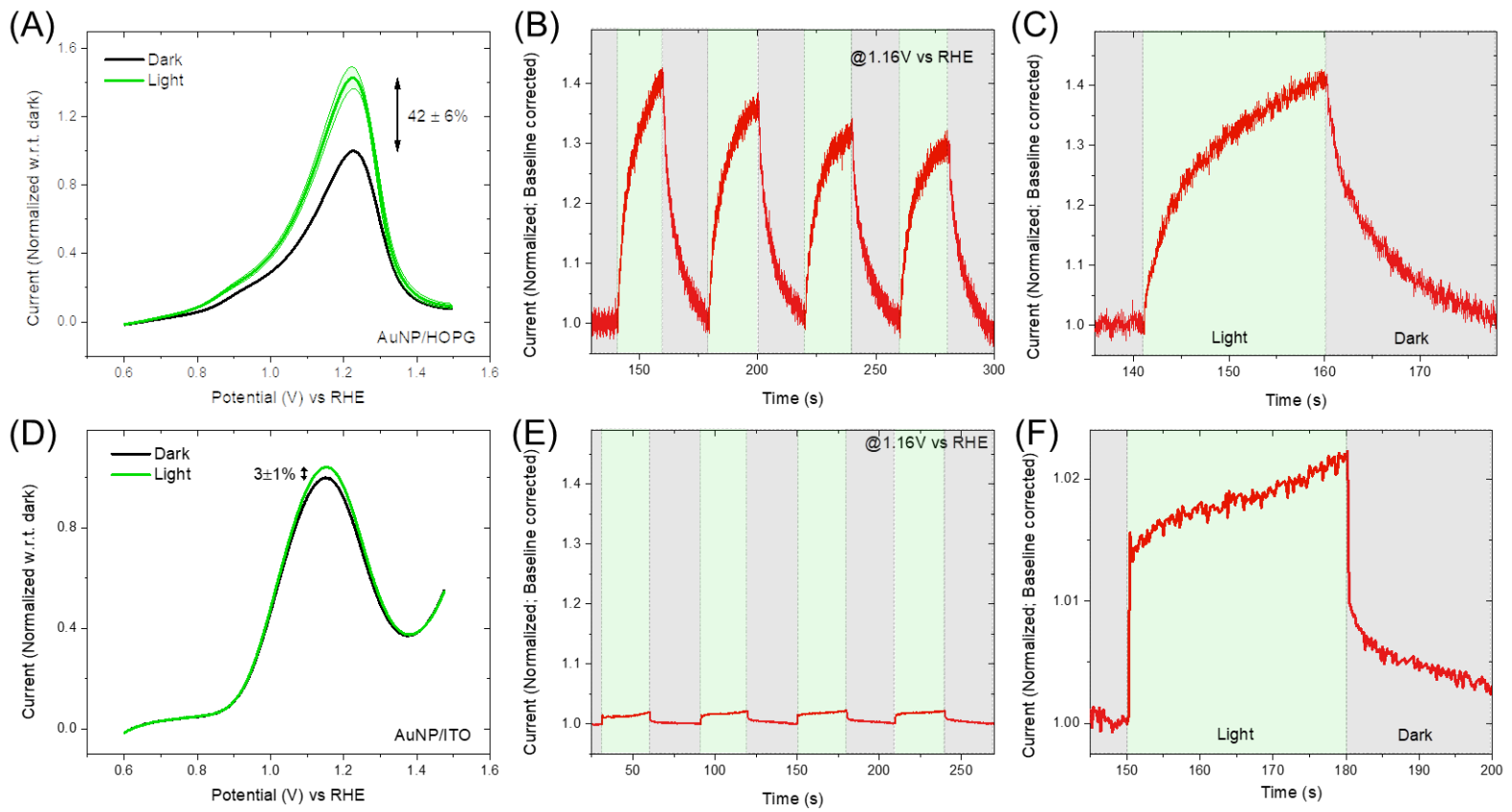

Figure 2: LSV scans on (A) AuNP/HOPG and (D) AuNP/ITO electrodes in $20 \mathrm{mM} \mathrm{BnOH}$ solution in the dark (black trace) and under illumination (green trace). Chronoamperometry measurements on (B-C) AuNP/HOPG and (E-F) AuNP/ITO electrodes in $20 \mathrm{mM} \mathrm{BnOH}$ solution under alternating illumination at a fixed applied potential of $1.16 \mathrm{~V}$ vs RHE. Illumination source: $532 \mathrm{~nm}$ laser $\left(325 \mathrm{~mW} / \mathrm{cm}^{2}\right)$. The voltammograms were recorded thrice to compute the mean values and the standard deviations. The standard deviations for currents recorded under illumination at different applied potentials are shown as a shaded region. The population mean value and the corresponding standard deviation are indicated.

Figures 2E-F displays photocurrent response of the AuNP/ITO electrode under alternating illumination (532 $\mathrm{nm}$ laser $\left.\left(325 \mathrm{~mW} / \mathrm{cm}^{2}\right)\right)$ in $20 \mathrm{mM} \mathrm{BnOH}$ solution. Similar to AuNP/HOPG electrodes, an increase and decay of the anodic photocurrent coincide with switching on (green 
areas in Figures 2E-F) and off (grey areas in Figures 2E-F) the light source. Interestingly, the nature of the current change is starkly different from the AuNP/HOPG electrodes as light on/off led to $\sim 1.5 \%$ instantaneous $(\sim 1 \mathrm{~s})$ rise/drop in the current followed by a slower change (Figures 2E-F). As the $\tau$ for the AuNP/ITO system was determined to be 870 milliseconds (Figure S4D), this instantaneous change can be attributed to the direct participation of hot carriers. It is important to emphasize that based on our analysis, hot carriers provide only $\sim 1.5 \%$ enhancement of the catalytic reaction.

\section{Temperature effects on AuNP/HOPG vs AuNP/ITO electrodes}

The significantly higher value (by $\sim 39 \%$ as suggested from Figure 2 A, D) of the photocurrent on the AuNP/HOPG in comparison to the AuNP/ITO electrodes attributed mainly to temperature effects invokes the question of whether the local heating due to the decay of hot carriers within AuNPs or HOPG itself, plays the key role in the photo-assisted enhancement. Since the temperature rise is proportional to the total amount of absorbed optical energy, ${ }^{24}$ the photocurrent response should reproduce the absorption spectrum of the material responsible for the heating. We performed excitation power and wavelength-dependent chronoamperometry measurements under alternating illumination (30 s light on/off) on the AuNP/HOPG electrodes in $20 \mathrm{mM} \mathrm{BnOH}$ solution. Figures $\mathbf{3 A}-\mathbf{C}$ show the dependence of the photocurrent on the excitation power for illumination with blue $(405 \mathrm{~nm}, \mathrm{~A})$, green $(532 \mathrm{~nm}, \mathrm{~B})$, and red $(650 \mathrm{~nm}$, C) lasers. The AuNP/HOPG electrodes displayed identical in shape photoresponses under illumination with the light sources of different wavelengths. To determine the values of the photocurrent at different wavelengths and intensities, the current recorded at the end of each dark period was used to create a baseline. After subtracting the baseline from the entire chronoamperometry data, the current recorded at $30 \mathrm{~s}$ of irradiation was considered as the photocurrent at a particular wavelength and intensity. The dependence of the photocurrent on the illumination intensity for different wavelengths is shown in Figure 3D. Photocurrent values 
at different intensities for different wavelengths can be fitted to a single line suggesting that there is no dependence of the photocurrent on the wavelength of the incident light. If the plasmonic effects on AuNPs were the primary source of the photocurrent, one would expect the photoresponse to be highly wavelength dependent with the enhancements coinciding with the LSP resonance features of the surface-adsorbed AuNPs (Figure 1B, grey trace). Since HOPG absorbs across the visible spectrum with a near-equal extinction coefficient, ${ }^{25,26}$ the wavelength independence of the photocurrent suggests that heating of the HOPG electrode itself due to absorption of the incident light is the primary source of the $\mathrm{BnOH}$ oxidation photo-enhancement on the AuNP/HOPG electrodes.
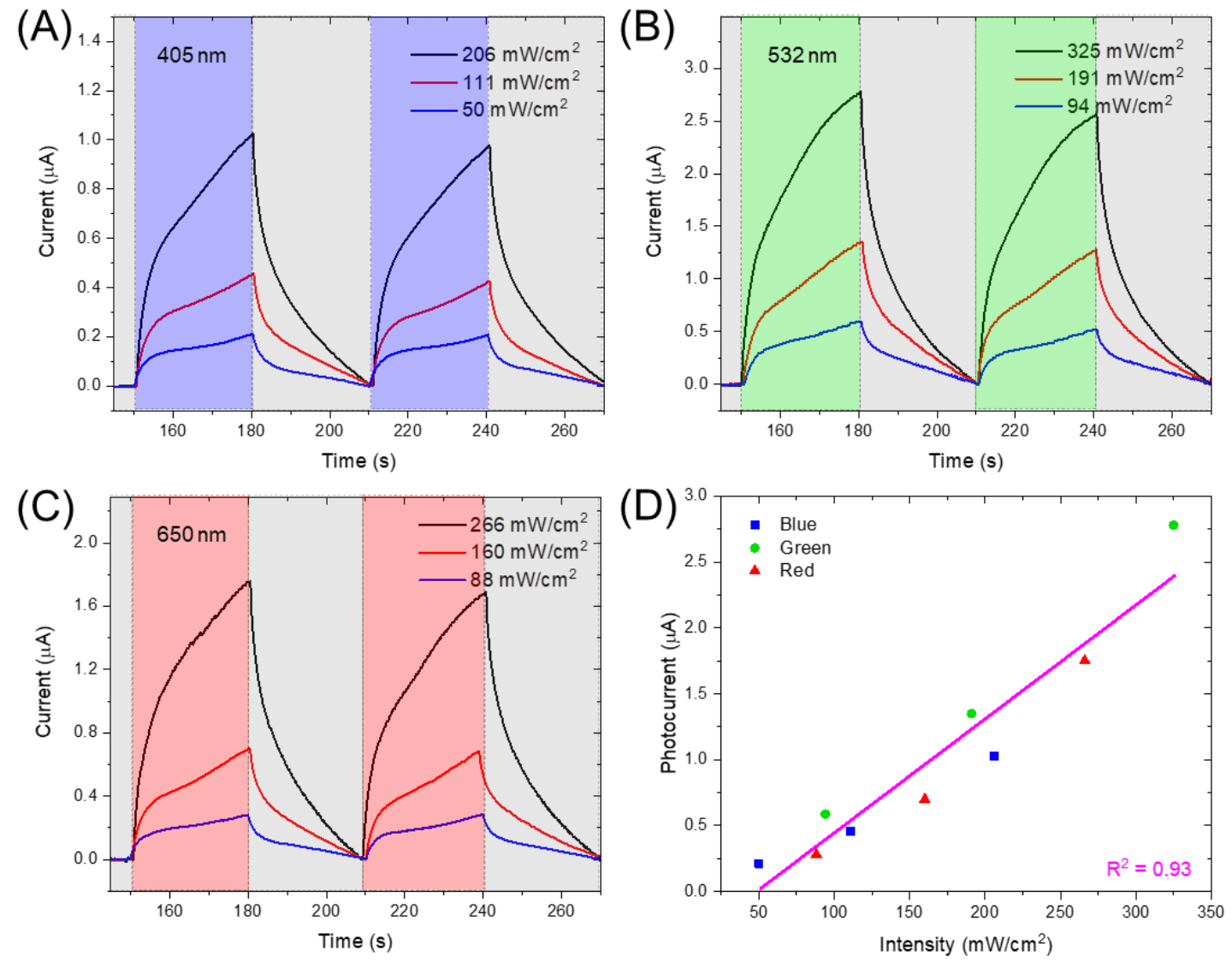

Figure 3: Chronoamperometry measurements on the AuNP/HOPG electrode in $20 \mathrm{mM} \mathrm{BnOH}$ solution at a fixed applied potential of $1.16 \mathrm{~V}$ vs RHE under alternating illumination of different intensities with (A) $405 \mathrm{~nm}$, (B) $532 \mathrm{~nm}$, and (C) $650 \mathrm{~nm}$ lasers. (D) Dependence of the photocurrent response on intensity at different wavelengths. 
To further confirm that the light absorption by the HOPG electrode results in the temperature increase, we compared the $\mathrm{CV}$ of the bare HOPG electrodes in buffer recorded under light illumination with the $532 \mathrm{~nm}\left(325 \mathrm{~mW} / \mathrm{cm}^{2}\right)$ laser (Figure S5A) with $\mathrm{CV}$ curves recorded in the dark at different temperatures of buffer solution varied externally using a thermostat (Figure S5B). As can be seen from a comparison of Figures S5A and B, increasing temperature leads to the similar changes in CV curves as the illumination. Specifically, an increase of cathodic current at $\sim 0.4 \mathrm{~V}$ vs RHE due to oxygen reduction reaction on the HOPG electrode and a decrease of the anodic current at $\sim 1.4 \mathrm{~V}$ vs RHE are observed upon illumination and with increasing temperature of buffer solution. A similar observation for an illuminated AuNP/HOPG electrode and its temperature-dependent response also confirms that the light absorption by HOPG is the primary source of the temperature increase (Supporting Note 1;

\section{Figure S6).}

To quantify the effective change in temperature due to irradiation, we performed $\mathrm{BnOH}$ electrooxidation on the AuNP/HOPG electrodes in the dark at various temperatures of solution controlled externally by a thermostat (Figure 4A). The measurements were repeated thrice at each temperature and statistical analysis was performed to obtain the average current and error values. The anodic peak current on the forward scan was found to increase linearly with the temperature (Figure 4B). Using the peak current dependence on the temperature as a calibration curve, the effective temperature increase upon irradiation with the $532 \mathrm{~nm}\left(325 \mathrm{~mW} / \mathrm{cm}^{2}\right)$ laser was found to be $5.3 \pm 0.8^{\circ} \mathrm{C}$. We further considered how the temperature increase affects the kinetics of $\mathrm{BnOH}$ electrooxidation.

\section{Mechanistic investigation of temperature effects on benzyl alcohol electrooxidation}

In plasmonic electrocatalysis literature, enhanced photocurrent at increased temperatures has generally been associated with higher diffusion of reactants and products at the catalyst/electrolyte interface due to significant local heating. ${ }^{17,27}$ The diffusion-controlled peak 
current $\left(i_{p}\right)$ for an irreversible electrochemical process, such as $\mathrm{BnOH}$ electrooxidation, can be described by the Delahay equation (Eqn. 2): ${ }^{28,29}$

$i_{p}=0 \cdot 282 n C_{b} A \sqrt{\frac{\Pi F \alpha n D v}{R T}}$

where $n$ is the number of electrons, $F$ is the Faraday's constant $(96485 \mathrm{C} / \mathrm{mol}), C_{b}$ is the bulk concentration of the reactant, $A$ is the surface area of the electrode, $D$ is the diffusion coefficient of the reactant, $R$ is the universal gas constant, $\alpha$ is the transfer coefficient, $v$ is the scan rate, and $T$ is the temperature. The equation predicts linear dependence of the $i_{p}$ on the square root of the $v$ and zero intercept for the $i_{p}$ (corrected for capacitive current contribution) vs $v^{1 / 2}$ line. Figures $\mathbf{S 7 A}$ and $\mathbf{C}$ show $\mathrm{CVs}$ of $\mathrm{BnOH}$ oxidation on the AuNP/HOPG electrodes at different $v$ in the dark and under illumination with the $532 \mathrm{~nm}\left(325 \mathrm{~mW} / \mathrm{cm}^{2}\right)$ laser, respectively. Though the $i_{p}$ vs $v^{1 / 2}$ relationship was linear (Figures S7B, D), the intercepts in both cases were nonzero, suggesting a mixed control of the peak current.
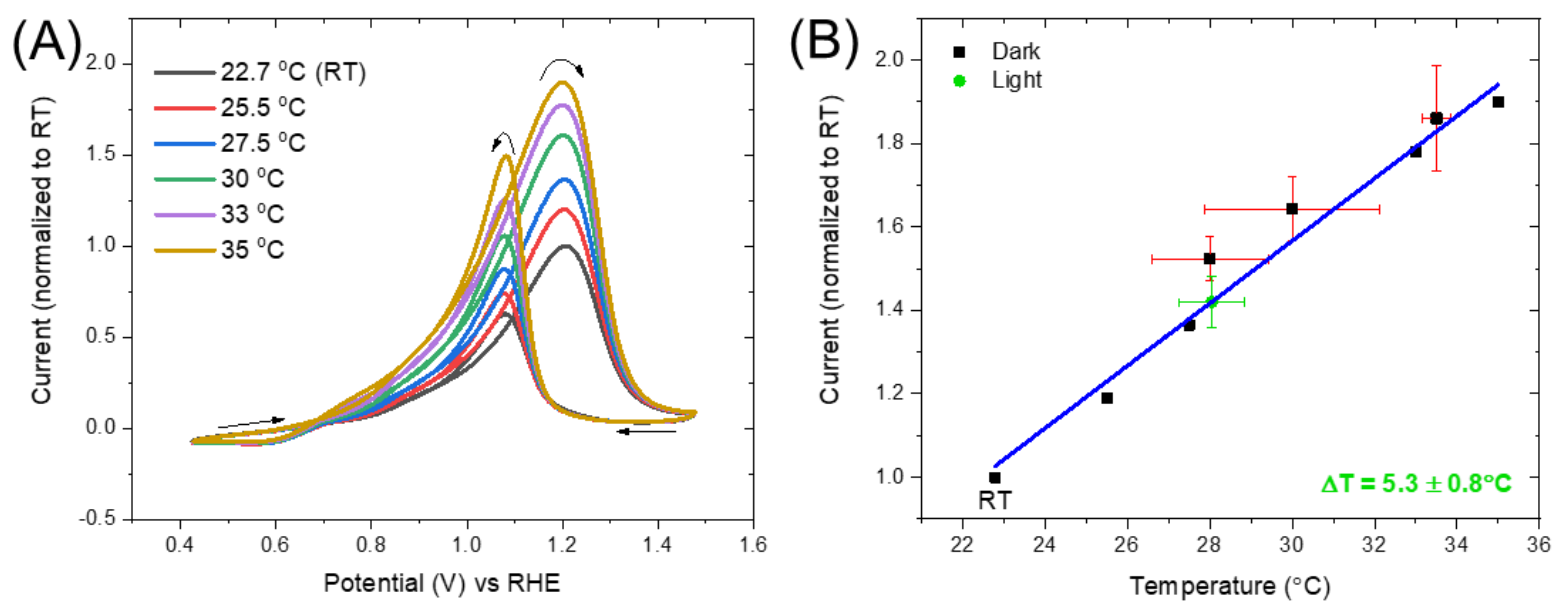

Figure 4: (A) CV scans (normalized to room temperature) on AuNP/HOPG electrodes in 20 $\mathrm{mM} \mathrm{BnOH}$ solution at different temperatures in the dark. (B) Determination of the temperature increase under irradiation $\left(532 \mathrm{~nm}, 325 \mathrm{~mW} / \mathrm{cm}^{2}\right.$; green point) from a calibration curve drawn using forward scan peak currents recorded at various temperatures. The voltammograms were 
recorded thrice to compute the population mean value and the corresponding standard deviation.

To probe the effect of mass transfer on the current, we performed hydrodynamic voltammetry measurements on rotating disk AuNP/HOPG electrodes (RDE) in $20 \mathrm{mM} \mathrm{BnOH}$ solution in the dark at varied rotation speeds $(0-2500 \mathrm{rpm})$. As expected for a mass-transport controlled reaction, the current increases with increasing the rotation speed. However, it does not reach a steady-state value even at high overpotentials (Figure 5A). In contrast to stationary electrodes, the mass transport of $\mathrm{BnOH}$ to the rotating electrode surface is both time and potential independent and proportional to the (electrode rotation speed $)^{1 / 2} \cdot{ }^{30}$ Therefore, for the masstransfer-limited process, one would reach a plateau current in RDE measurements once the mass transport of the analyte limits the electrochemical reaction. The peak formation on the AuNP/HOPG electrode in RDE experiments confirms that the electrode process at potentials corresponding to the peak current on the stationary $\mathrm{CV}$ is not entirely controlled by diffusion.
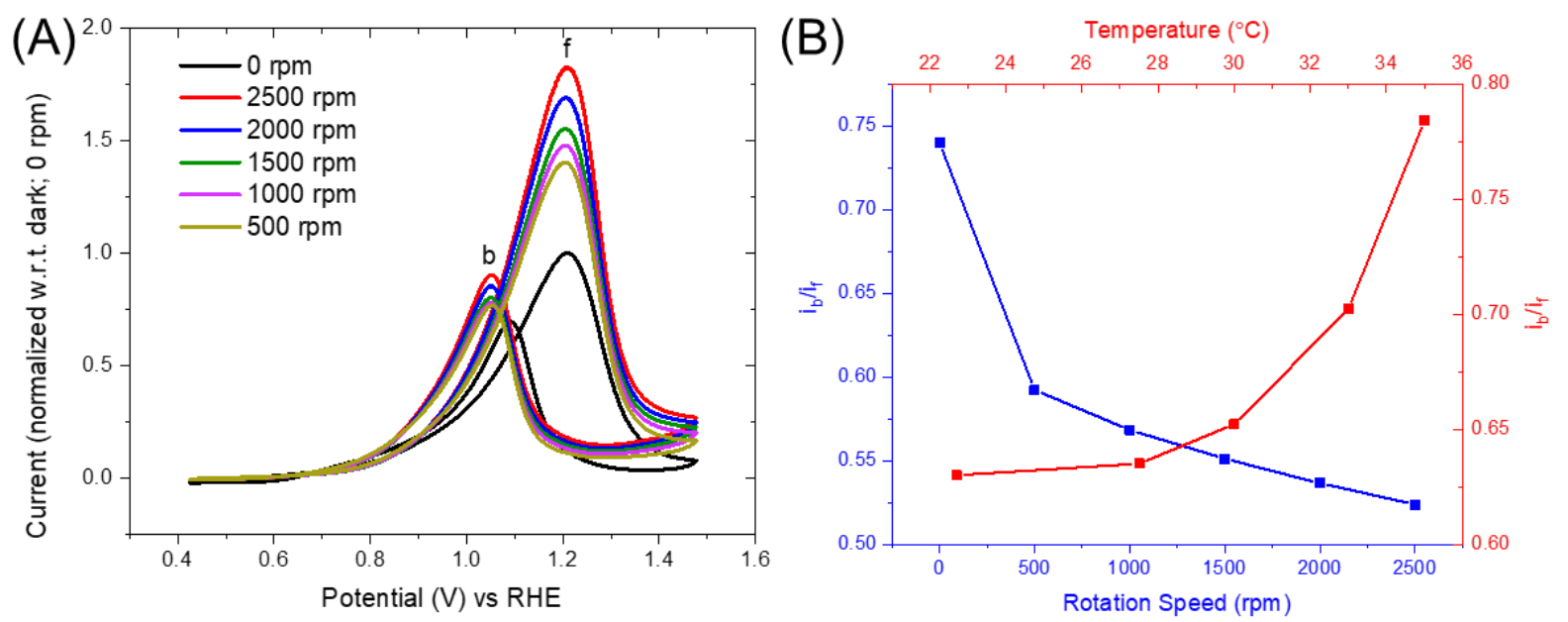

Figure 5: (A) CV scans on AuNP/HOPG electrodes in $20 \mathrm{mM} \mathrm{BnOH}$ solution at different rotation speeds in the dark. (B) Dependence of the backward scan peak current $\left(i_{b}\right) /$ forward scan peak current $\left(i_{f}\right)$ ratio determined from the $\mathrm{CV}$ curves on temperature (red trace) and rotation speeds (blue trace). 
By comparing CVs of the AuNP/HOPG electrodes in buffer without and with $20 \mathrm{mM} \mathrm{BnOH}$ (Figure S8), we found that the anodic peak corresponding to $\mathrm{BnOH}$ oxidation on the forward scan $(f)$ coincides with the commencement of $\mathrm{Au}$ oxidation into $\mathrm{AuO}_{\mathrm{x} \cdot}{ }^{16,31}$ Interestingly, as $\mathrm{AuO}_{\mathrm{x}}$ is reduced back to $\mathrm{Au}$ on the backward scan (b), the reemergence of the $\mathrm{BnOH}$ electrooxidation current is observed. This suggests that the oxidation of $\mathrm{AuO}_{\mathrm{x}}$ leads to loss of the catalytically active Au surface resulting in the peak formation on the forward scan.

The mixed-controlled nature of the peak current, as was observed for $\mathrm{BnOH}$ electrooxidation on the AuNP/HOPG surface, implies that the electrocatalytic activity can increase if either diffusion or charge transfer rates or both increase. To probe the effect of irradiation on diffusion rates, we performed $\mathrm{CV}$ measurements on the AuNP/HOPG electrodes in $1 \mathrm{mM}$ solution of $\mathrm{Ru}\left(\mathrm{NH}_{3}\right)_{6} \mathrm{Cl}_{2}$ in $1 \mathrm{M} \mathrm{KCl}$ in the dark and under illumination with the $532 \mathrm{~nm}\left(325 \mathrm{~mW} / \mathrm{cm}^{2}\right)$ laser (Figure S9A). $\mathrm{Ru}\left(\mathrm{NH}_{3}\right)_{6} \mathrm{Cl}_{2}$ is a reversible redox probe with fast electrode kinetics and the CV peak currents entirely controlled by mass transport (diffusion). ${ }^{22,32}$. Therefore, the change in the peak current can provide a quantitative estimate of diffusion enhancement due to heating upon irradiation. As can be seen from Figure $\mathbf{S 9 A}$, the peak currents of $\mathrm{Ru}\left(\mathrm{NH}_{3}\right)_{6} \mathrm{Cl}_{2}$ increased by only $1.6 \%$ under irradiation. A similar response was observed in CVs recorded in the dark at increasing temperatures of solution (Figure S9B, C). These results demonstrate that the increased rate of diffusion due to heating cannot explain the $\sim 42 \%$ photocurrent enhancement observed for $\mathrm{BnOH}$ oxidation on the AuNP/HOPG electrodes.

As the enhanced diffusion due to local heating was found not to considerably increase the peak current, facilitated charge transfer at higher temperatures should be responsible for improved electrocatalysis. While the RDE measurements are affected by the Au surface oxidation, they can still be used to identify the current range, where the catalytic activity is entirely charge transfer controlled. ${ }^{30}$ In an RDE measurement, any current enhancement with increasing the rotation speed arises only from the increased mass transport. Thus, the current range that 
remains unchanged with increasing the rotation speed is representative of a region, entirely controlled by charge transfer kinetics. For the system under study, this current range was found to be $0.018-0.1 \mu \mathrm{A}$. We identified the potential window corresponding to this current range under irradiation from Figure 2A. Its comparison with the current recorded in the dark in the same potential window reveals a $(45 \pm 3.3) \%$ increase of the charge transfer rate under illumination (Figures 6A-B). This enhancement of current is the same within an error to the $(42 \pm 6) \%$ current enhancement on the forward peak in CV under illumination. We would like to mention that as a rule of thumb, the Arrhenius relationship predicts doubling of a reaction rate with each $10{ }^{\circ} \mathrm{C}$ increase in temperature. Therefore, our observation of $(45 \pm 3.3) \%$ increase of the charge transfer rate with a temperature increase of $\sim 5.3{ }^{\circ} \mathrm{C}$ arising from illumination is consistent with this prediction. Thus, the increased temperature resulting from light absorption by HOPG facilitates charge transfer kinetics at the electrode-substrate interface. This is further supported by the opposite behavior of the ratio between the backward peak current $\left(i_{b}\right)$ and the forward peak current $\left(i_{f}\right)$ for $\mathrm{BnOH}$ oxidation under the temperature variations vs the rotation speed variation in the RDE measurements (Figure 5B). The $i_{b} / i_{f}$ ratio decreased with increasing the rotation speed, as the rotation speed affects only mass transport and the effect of mass transport limitation is higher on $i_{f}$ compared to $i_{b}$. In contrast, $i_{b} / i_{f}$ increased with increasing temperature. This is because $i_{b}$ is more influenced by charge transfer kinetics than $i_{f}$ and therefore, while increased temperature led to an increase in both peaks, its effect on $i_{b}$ was higher compared to $i_{f}$. 

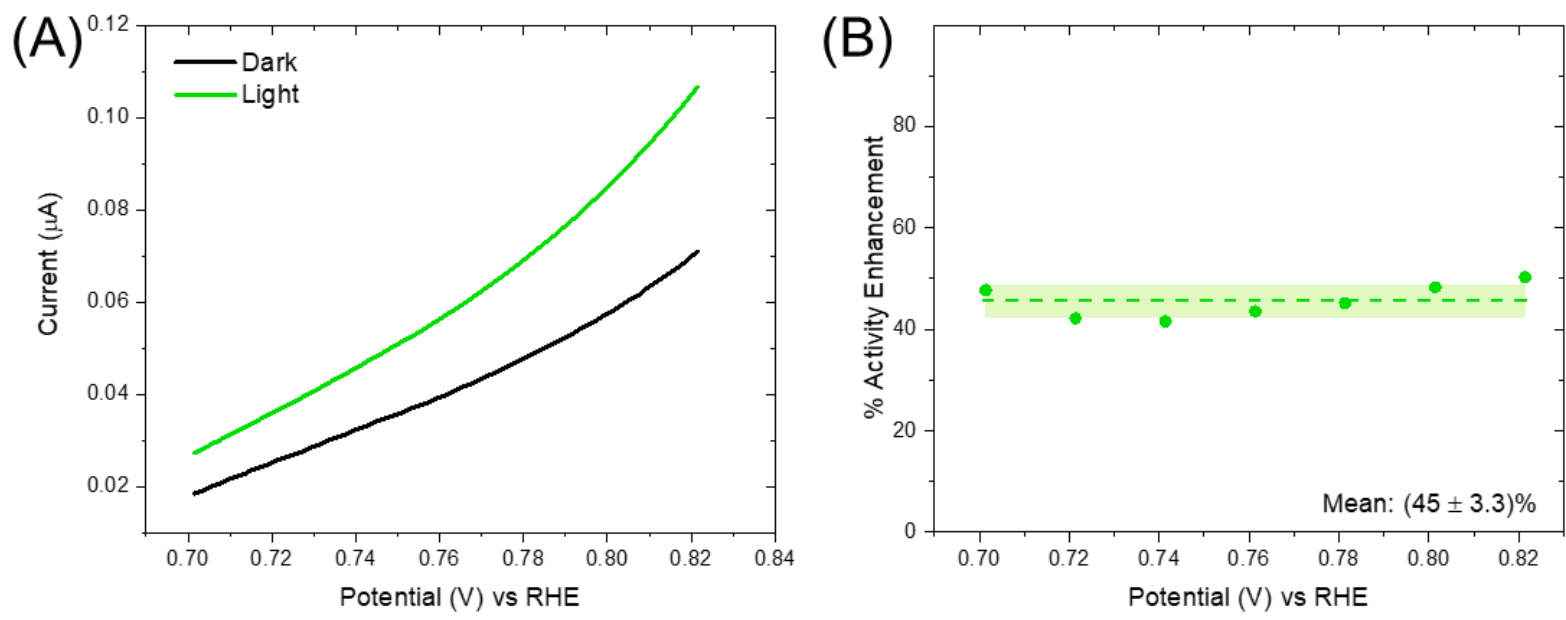

Figure 6: (A) LSV curves on AuNP/HOPG electrodes in $20 \mathrm{mM}$ BnOH solution in the potential region controlled only by charge transfer kinetics (as determined from RDE experiments) in the dark and under illumination (532 nm laser, $325 \mathrm{~mW} / \mathrm{cm}^{2}$ ), and (B) The photoinduced electrocatalytic activity enhancement determined from (A) as a ratio of current under illumination to the dark current. The voltammograms were recorded thrice to compute the mean values and corresponding standard deviations. The standard deviations at different potentials are shown as a shaded region. The population mean value and the corresponding standard deviation are indicated.

\section{Conclusion}

We have carried out a systematic investigation of the role of the inert electrode support in photoelectrocatalysis on plasmonic AuNPs. We have noticed a striking difference in the photocurrent responses for AuNPs supported on HOPG and ITO electrodes. The photocurrent on the AuNP/ITO electrodes was 14 times lower than on the AuNP/HOPG electrodes and consisted of two components: a fast-decaying upon switching of illumination component and a slow-decaying component, while the photoresponse of the AuNP/HOPG electrodes had only a slow-decaying component. We suggested using the cell constant $\left(\mathrm{R}_{\mathrm{S}} \mathrm{C}_{\mathrm{dl}}\right)$ as a quantitative parameter to differentiate between the contribution of hot carriers vs temperature to the photoresponse in plasmon-driven electrocatalysis based on their different timescales. The 
photoresponse with a timescale greater than the cell constant could be attributed to temperature effects, while the response comparable in time with the cell constant arises from hot carriers. We found that hot carriers have a negligible contribution to the increased $\mathrm{BnOH}$ electrooxidation on AuNPs loaded on the HOPG surface under illumination, and the observed photocurrent resulted from increased temperature. Moreover, we demonstrated that light absorption by the HOPG substrate and not the recombination of hot carriers in AuNPs is responsible for the temperature increase. Finally, in contrast to previous reports where the effect of increased temperature has only been attributed to higher diffusion of the substrate in the vicinity of the electrode, we showed that the enhancement of catalysis can arise from the increased charge transfer kinetics at elevated temperatures.

This work demonstrated that despite being catalytically inert, carbon-based support materials under illumination can significantly stimulate the electrocatalytic activity of catalytic centers placed on its surface by virtue of their wide spectral absorption. We would like to emphasize that being catalytically inert may not be enough to be chosen as the electrode support for evaluating the true photocatalytic activity of an immobilized catalyst under illumination.

\section{Author Information}

Corresponding author e-mail: alina.sekretareva@kemi.uu.se

\section{Declaration of competing interests}

The authors declare no financial interests.

\section{Acknowledgments}

A.S. thanks FORMAS (N 2019-01126) for supporting this research. A.S. and S.G. acknowledge support from Carl Tryggers Stiftelse (N CTS 19:326). A.S. and S.G. also thank Petko Chernev for his help with cell design for electrochemical measurements. 


\section{References}

Lang, X.; Chen, X.; Zhao, J. Heterogeneous Visible Light Photocatalysis for Selective Organic Transformations. Chem. Soc. Rev. 2013, 43 (1), 473-486. https://doi.org/10.1039/C3CS60188A.

(2) Kamarudheen, R.; Aalbers, G. J. W.; Hamans, R. F.; Kamp, L. P. J.; Baldi, A. Distinguishing Among All Possible Activation Mechanisms of a Plasmon-Driven Chemical Reaction. ACS Energy Lett. 2020, $5 \quad$ (8), 2605-2613. https://doi.org/10.1021/acsenergylett.0c00989.

(3) Yu, S.; Wilson, A. J.; Kumari, G.; Zhang, X.; Jain, P. K. Opportunities and Challenges of Solar-Energy-Driven Carbon Dioxide to Fuel Conversion with Plasmonic Catalysts. ACS Energy Lett. 2017, 2, 2058-2070. https://doi.org/10.1021/acsenergylett.7b00640.

(4) Zhang, Y.; He, S.; Guo, W.; Hu, Y.; Huang, J.; Mulcahy, J. R.; Wei, W. D. Surface-Plasmon-Driven Hot Electron Photochemistry. Chem. Rev. 2018, 118 (6), 2927-2954. https://doi.org/10.1021/acs.chemrev.7b00430.

(5) Zhao, J.; Xue, S.; Ji, R.; Li, B.; Li, J. Localized Surface Plasmon Resonance for Enhanced Electrocatalysis. Chem. Soc. Rev. 2021, 50 (21), 12070-12097. https://doi.org/10.1039/D1CS00237F.

(6) Chen, D.; Zhang, R.; Wang, R.; Negro, L. D.; Minteer, S. D. Gold NanofiberBased Electrodes for Plasmon-Enhanced Electrocatalysis. J. Electrochem. Soc. 2016, 163 (14), H1132. https://doi.org/10.1149/2.0501614jes.

(7) Robatjazi, H.; Bahauddin, S. M.; Doiron, C.; Thomann, I. Direct Plasmon-Driven $\begin{array}{llllll}\text { Photoelectrocatalysis. Nano } & \text { Lett. 2015, } 15 & \text { (9), 6155-6161. }\end{array}$ https://doi.org/10.1021/acs.nanolett.5b02453.

(8) Shi, F.; He, J.; Zhang, B.; Peng, J.; Ma, Y.; Chen, W.; Li, F.; Qin, Y.; Liu, Y.; Shang, W.; Tao, P.; Song, C.; Deng, T.; Qian, X.; Ye, J.; Wu, J. Plasmonic-Enhanced Oxygen Reduction Reaction of Silver/Graphene Electrocatalysts. Nano Lett. 2019, 19 (2), 1371-1378. https://doi.org/10.1021/acs.nanolett.8b05053.

(9) Liao, X.-W.; Wang, S.-S.; Xu, G.-Y.; Wang, C. Enhanced Electrocatalysis via Boosted Separation of Hot Charge Carriers of Plasmonic Gold Nanoparticles Deposited on Reduced Graphene Oxide. ChemElectroChem 2019, 6 (5), 1419-1426. https://doi.org/10.1002/celc.201801683.

Lee, J.-E.; Marques Mota, F.; Choi, C. H.; Lu, Y.-R.; Boppella, R.; Dong, C.-L.; Liu, R.-S.; Kim, D. H. Plasmon-Enhanced Electrocatalytic Properties of Rationally Designed Hybrid Nanostructures at a Catalytic Interface. Adv. Mater. Interfaces 2019, 6 (2), 1801144. https://doi.org/10.1002/admi.201801144.

Pumera, M. Graphene-Based Nanomaterials and Their Electrochemistry. Chem. Soc. Rev. 2010, 39 (11), 4146-4157. https://doi.org/10.1039/C002690P. 

Pretty Gold: Noble Metal Surface Plasmon Resonance and Its Enhancement of the Radiative and Nonradiative Properties of Nanocrystals of Different Shapes. Chem. Soc. Rev. 2006, 35 (3), 209-217. https://doi.org/10.1039/B514191E.

Balamurugan, B.; Maruyama, T. Evidence of an Enhanced Interband Absorption in Au Nanoparticles: Size-Dependent Electronic Structure and Optical Properties. Appl. Phys. Lett. 2005, 87 (14), 143105. https://doi.org/10.1063/1.2077834.

Scheeler, S. P.; Mühlig, S.; Rockstuhl, C.; Hasan, S. B.; Ullrich, S.; Neubrech, F.; Kudera, S.; Pacholski, C. Plasmon Coupling in Self-Assembled Gold Nanoparticle-Based Honeycomb Islands. J. Phys. Chem. C 2013, 117 (36), 18634-18641. https://doi.org/10.1021/JP405560T.

Xi, C.; Marina, P. F.; Xia, H.; Wang, D. Directed Self-Assembly of Gold Nanoparticles into Plasmonic Chains. Soft Matter 2015, 11 (23), 4562-4571. https://doi.org/10.1039/C5SM00900F.

(16) Ureta-Zañartu, M. S.; Berríos, C.; González, T.; Fernández, F.; Báez, D.; Salazar, R.; Gutiérrez, C. Electrocatalytic Oxidation of Alcohols at Gold Electrodes in Alkaline Media. Int. J. Electrochem. Sci. 2012, 7 (9), 8905-8928.

Wang, C.; Nie, X.-G.; Shi, Y.; Zhou, Y.; Xu, J.-J.; Xia, X.-H.; Chen, H.-Y. Direct Plasmon-Accelerated Electrochemical Reaction on Gold Nanoparticles. ACS Nano 2017, 11 (6), 5897-5905. https://doi.org/10.1021/acsnano.7b01637.

Sui, N.; Gao, H.; Zhu, J.; Jiang, H.; Bai, Q.; Xiao, H.; Liu, M.; Wang, L.; Yu, W. W. Shape- and Size-Dependences of Gold Nanostructures on the Electrooxidation of Methanol under Visible Light Irradiation. Nanoscale 2019, 11 (39), 18320-18328. https://doi.org/10.1039/C9NR06839B.

(19) Huang, L.; Zou, J.; Ye, J.-Y.; Zhou, Z.-Y.; Lin, Z.; Kang, X.; Jain, P. K.; Chen, S. Synergy between Plasmonic and Electrocatalytic Activation of Methanol Oxidation on Palladium-Silver Alloy Nanotubes. Angew. Chem. Int. Ed. 2019, 58 (26), 8794-8798. https://doi.org/10.1002/anie.201903290.

Jain, P. K. Taking the Heat off of Plasmonic Chemistry. J. Phys. Chem. C 2019, 123 (40), 24347-24351. https://doi.org/10.1021/acs.jpcc.9b08143.

(21) Shahbazyan, T. V. Landau Damping of Surface Plasmons in Metal $\begin{array}{lllllll}\text { Nanostructures. } & \text { Phys. } & \text { Rev. } & B & \text { 2016, } & 94 & \text { (23), }\end{array} 235431$. https://doi.org/10.1103/PhysRevB.94.235431.

(22) Bard, A. J.; Faulkner, L. R. Electrochemical Methods : Fundamentals and Applications, 2nd ed.; John Wiley \& Sins, INC.

Rieth, A. J.; Gonzalez, M. I.; Kudisch, B.; Nava, M.; Nocera, D. G. How Radical Are "Radical" Photocatalysts? A Closed-Shell Meisenheimer Complex Is Identified as a SuperReducing Photoreagent. J. Am. Chem. Soc. 2021, 143 (35), 14352-14359. https://doi.org/10.1021/jacs.1c06844. 

Nanoparticles Induced by Light Emitting Diodes. Appl. Therm. Eng. 2016, 99, 1093-1100. https://doi.org/10.1016/J.APPLTHERMALENG.2016.01.077.

Wang, S.; Wang, C.; Ji, X. Towards Understanding the Salt-Intercalation Exfoliation of Graphite into Graphene. RSC Adv. 2017, 7 (82), 52252-52260. https://doi.org/10.1039/C7RA07489A.

Dovbeshko, G. I.; Romanyuk, V. R.; Pidgirnyi, D. V.; Cherepanov, V. V.; Andreev, E. O.; Levin, V. M.; Kuzhir, P. P.; Kaplas, T.; Svirko, Y. P. Optical Properties of Pyrolytic Carbon Films Versus Graphite and Graphene. Nanoscale Res. Lett. 2015, 10 (1), 234. https://doi.org/10.1186/s11671-015-0946-8.

Yu, Y.; Sundaresan, V.; Willets, K. A. Hot Carriers versus Thermal Effects: Resolving the Enhancement Mechanisms for Plasmon-Mediated Photoelectrochemical Reactions. J. Phys. Chem. C 2018, 122 (9), 5040-5048. https://doi.org/10.1021/acs.jpcc.7b12080.

(28) Kishioka, S.; Yamada, A. Electrooxidation of Vanillyl Alcohol in Acidic Aqueous Solution Using Rotating Ring-Disk Electrode Voltammetry. Anal. Sci. 2005, 21 (4), 429-432. https://doi.org/10.2116/analsci.21.429.

(29) Delahay, P.; Paul Delahay, B. Theory of Irreversible Waves in Oscillographic Polarography. 1952.

(30) Gileadi, E. Physical Electrochemistry- Fundamentals, Techniques and Applications, 2nd ed.; Wiley-VCH Verlag GmbH \& Co., 2013.

(31) Rodriguez, P.; Koper, M. T. M. Electrocatalysis on Gold. Phys. Chem. Chem. Phys. 2014, 16 (27), 13583-13594. https://doi.org/10.1039/c4cp00394b.

Limon-Petersen, J. G.; Han, J. T.; Rees, N. V.; Dickinson, E. J. F.; Streeter, I.; Compton, R. G. Quantitative Voltammetry in Weakly Supported Media. Chronoamperometric Studies on Diverse One Electron Redox Couples Containing Various Charged Species: Dissecting Diffusional and Migrational Contributions and Assessing the Breakdown of Electroneutrality. J. Phys. Chem. C 2010, 114 (5), 2227-2236. https://doi.org/10.1021/jp9097149. 